\title{
Designing heterocyclic selective kinase inhibitors: from concept to new drug candidates
}

\author{
Serge Halazy \\ Serono Pharmaceutical Research Institute, 14, chemin des Aulx 1228 Plan-Les-Ouates \\ Geneva, Switzerland \\ E-mail: serge.halazy@serono.com
}

\begin{abstract}
Kinases represent one of the most popular and promising target class in drug discovery. Success in finding new therapeutics will depend on the validation of the kinase choosen with respect to the disease of interest, and on the ability of chemists to design and synthesize inhibitors which are selective for this particular kinase. One of the most powerful validation tool for kinases is the Analogue-Sensitive Kinase Allele ( ASKA) technology, where chemists and biologists have engineered modified kinases and inhibitors by generating functionally active kinase mutants which are specifically inhibited by a chemically modified inhibitor, thus allowing to study specific responses in knock-in animals. The design of selective, ATP competitive kinase inhibitors has been succesfully achieved by combining different technologies like cpomputational chemistry, structure-based design and combinatorial chemistry as illustrated by the discovery of AS602801, a potent and selective JNK inhibitor with therapeutic potential in MS and fibrosis. The exploration of non ATP-competitive inhibitors is also very promising, as illustrated by the case of MEK inhibitors which show strong interest as anti-cancer agents. In the future, the rationale design of selective kinase inhibitors will move to the design of compounds that will induce a selective perturbation of cell regulation, where kinases play a critical role.
\end{abstract}

Keywords: Kinases, ASKA, JNK, MEK, drug discovery, signal transduction, chemical kinomics

\section{Contents}

Introduction

1. Kinases validation: the power of ASKA technology

2. Designing selective kinase inhibitors

3. Taking advantage of lack of selectivity

4. Non-ATP competitive inhibitors

5. Kinases at the crossroad of complex signalling pathways 


\section{Introduction}

The first wave of molecular medicines has successfully been driven by drug discovery efforts targeting almost exclusively proteins which are physiologically modulated, activated, inhibited or recognized by small molecules or peptides. Cholesterol biosynthesis inhibitors, anti-cancer anti-metabolites targeting DNA synthesis enzymes or agonists/antagonists of GPCR's activated by neuro-amines (serotonin, histamine, nor-adrenalin, ...) are well-known examples which have led to important new drugs in the last 30 years. During that period of time, progresses in cell biology, molecular biology and structural biology, and more recently in new emerging areas like genomics, proteomics or human genetics (in parallel to the human genome sequencing) have identified a large number of new functional proteins as potential drug targets. These proteins don't bind small molecules or peptides in their physiological role, but display their critical function essentially through conformational changes and interactions with other proteins, either inside or outside the cell. The second wave of molecular medicines will focus on this class of protein targets and this represents a serious challenge for small molecule drug design, since chemists master the art of designing modulators of protein/small ligand interactions, (the first wave of molecular medicine) but have not been very successful so far in designing modulators of protein-protein interactions.

This is where kinases show-up as one of the most important target class under investigation in drug discovery these days; ${ }^{1}$ protein conformation state (and therefore the ability of said protein to interact with other proteins) is very often dependent on its phosphorylation state, which is controlled by intracellular kinases. Moreover, kinases are enzymes which bind ATP from which the phosphate group will be transferred to the protein substrate. This is exactly why kinases are so exciting in drug discovery: they act as a molecular switch which controls intracellular protein-protein interactions (the targets of the second wave of molecular medicine) and are at the same time potentially "druggable" in the sense that they bind a small molecule (ATP), making them feasible targets for medicinal chemistry.

Since the pioneering work ${ }^{2}$ of Ed Fisher and Ed Krebs (who were awarded the Nobel prize in 1992), the reversible phosphorylation of proteins has been widely recognised as central to the regulation of most aspects of cell function and cell physiology. Increasing numbers of human diseases are known to involve mutations, over-expression, genetic association or malfunctioning of protein kinases and phosphatases, and their regulators and effectors. Signal Transduction, mediated by protein phosphorylation is extremely complex and our understanding of signalling mechanisms and their implication in diseases is still in its infancy. However, it represents a fantastic field of investigation to explore where small molecules will play a critical role, both in helping understand cell biology mechanisms to gain knowledge and progress, but also, hopefully in proposing therapeutic solutions to unmet medical needs.

Inhibition of kinases (which, most of the time positively relay signalling events) is, a powerful way to inhibit or control signal transduction cascades, which become particularly relevant in drug discovery when pathological conditions are associated with stimulation of intra- 
cellular signalling. ${ }^{3,4}$ This is the case in important therapeutic areas like cancer, inflammation and apoptosis-related disorders where efforts to find selective kinase inhibitors have literally exploded in the last 5 years. ${ }^{5}$ A landmark event occurred in May 2001 when Gleevec, the first important drug targeting specifically a protein kinase (ABL) was approved for clinical use to treat chronic myeloid leukaemia. ${ }^{6}$

The human genome encodes for around 500 different kinases, ${ }^{7}$ which control most of cellular events, directly or indirectly. Chemical kinomics may be defined as the description of all possible inhibitors against all kinases. From a therapeutic perspective efficient application of chemical kinomics will have to address 2 important pertinent questions: What kinase for what disease? And how to design selective inhibitors for specific kinases of therapeutic interest?

\section{Kinases validation: the power of ASKA technology}

Validation of individual kinases for drug discovery (which means to get enough convincing evidence that inhibitors of such kinases will demonstrate a therapeutic effect in patients during phase II clinical trials) is not trivial and remains one of the most challenging aspects of postgenomic drug discovery. Dissection of signal transduction mechanisms is certainly a good starting point, generally followed by knock-out animal experiments and/or pharmacological studies with known inhibitors. However, none of these approaches is fully satisfactory and new ones are currently under investigation based on chemical genetics. For example, the AnalogueSensitive Kinase Allele (ASKA) technology, ${ }^{8}$ where chemists and biologists have engineered modified kinases and inhibitors (by generating functionally active kinase mutants which are specifically inhibited by a chemically modified inhibitor), allows one to study specific responses in vivo in knock-in animals, (and therefore validate the kinase for a given disease). The strategy involves a point mutation to create a unique pocket in the ATP binding site of the kinase of interest; a specific inhibitor of the engineered kinase has been synthesized by derivatizing a known kinase inhibitor with a bulky group designed to fit the novel active site pocket. This has been initially illustrated ${ }^{9}$ with v-Src kinase which has been rationally engineered (I338G v-Src or Src ASKA) to accommodate a highly potent and uniquely selective inhibitor (1NaPP1; 4-amino1-tert-butyl-3-(1'-naphthyl)pyrazolo [3,4.d] pyrimidine) which has been designed by rationale derivation of PP1, a known Src inhibitor (Fig. 1). 


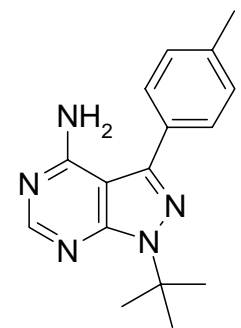

PP1

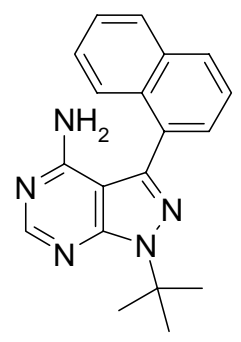

1-NaPP1

IC50 ( Src-wt) : $1 \mu \mathrm{M}$

IC50 ( Src-ASKA) : 1,5 nM

\section{Figure 1}

1-NaPP1 potently inhibits Src-ASKA with a high degree of selectivity versus any wildtype kinase. Importantly, 1-NaPP1 also potently inhibits ASKAs that have been generated from multiple kinases throughout the kinome. The consequence is that 1-NaPP1 strongly inhibits the engineered kinase (ASKA) in whole cells or in animals where it has replaced the wild-type enzyme without inhibiting phosphorylation in cells/animals that only express wild-type kinases. This offers a powerful tool to evaluate the role of the engineered kinase in disease models, in a physiological and pharmacological relevant manner.

The beauty of this approach lies in the fact that it can be applied to a large number of tyrosine and serine/threonine kinases (cytoplasmic and membrane integrated) where the same type of mutation can take place, (based on ATP binding site sequence alignment) rendering the mutant kinase (ASKA) sensitive to inhibition by 1-NaPP1, thus offering a "quasi-universal" tool for kinase validation. The other important point to underline is that the mutation in ASKA is most of the time silent in terms of kinase function (i.e. catalytic activity, substrate specificity, regulation, cell distribution, ...). 1-NaPP1 is cell permeable and has excellent bioavailability, allowing to treat ASKA cells or whole animals to study genomic, proteomic, cellular, physiologic and/or phenotypic consequences of such inhibition of the ASKA target. Such mouse disease models with functionally intact, specifically and pharmacologically inhibitable kinase targets will also provide invaluable information with respect to biomarkes discovery, therapeutic index determination, and profiling of lead compounds to determine target-based vs. compoundbased side effects. A complimentary technology, known as P-target, utilizes the same ASKA

mutants to tag the direct substrates of kinase with a unique "bumped" ATP-analog. ${ }^{10,11}$ P-target allows direct kinase substrate identification (pathway mapping) by using functional ATP analogues that are uniquely accepted as substrates by the ASKA as it is the case of 6-N-benzyl ATP (Fig. 2). When incubating cell lysates with a radiolabeled 6-N-benzyl ATP, only direct substrates of the mutated kinase (and nothing else) become radiolabeled, therefore allowing their rapid identification. P-target allows identification of direct substrates of kinases (since it can be 
applied to a vast majority of kinases, exactly as the 1-NaPP1 inhibition described above) giving access to new target opportunities downstream of kinases and new access for biomarkers.

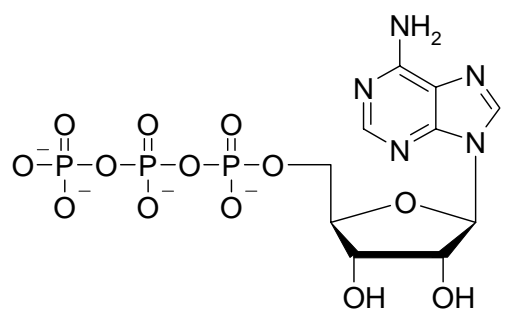

ATP

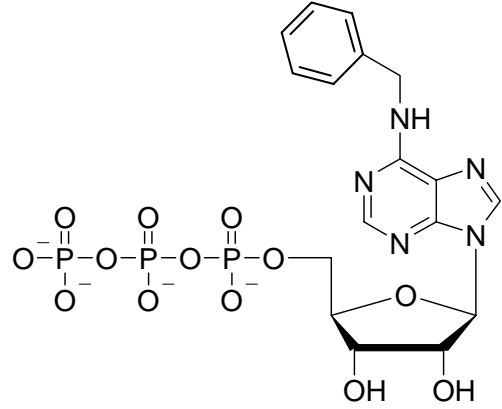

6-N-benzyl ATP

Figure 2

\section{Designing selective kinase inhibitors}

Beyond the choice and validation of the proper kinase as target for drug discovery, the next challenge will be the design of selective inhibitors. So far, the vast majority of kinase inhibitors reported to date are ATP competitive, which means that they bind to the ATP binding site, which is common for all kinases and which is highly conserved in terms of amino acid sequence.

It is therefore not surprising that plenty of ATP-competitive inhibitors are poorly selective (as for example staurosporine). As part of our program directed toward the discovery of JNK inhibitors in inflammation/apoptosis related disorders, we have discovered a new chemical class of ATP-competitive inhibitors derived from the benzothiazole scaffold. ${ }^{12,13}$ One of the first compound from this series (AS601245) which was identified as a potent JNK inhibitor also displayed strong activity against a few other kinases, as shown by the selectivity profile depicted in chart A below (Fig. 3).

Chemical modifications based on JNK 3D-structure, docking experiments (Fig. 4) and inhouse SAR data provided second generation JNK inhibitors, which were found highly selective versus other kinases, as shown by the selectivity profile of AS602801 depicted in chart B, (Fig. 3). 
Chart A
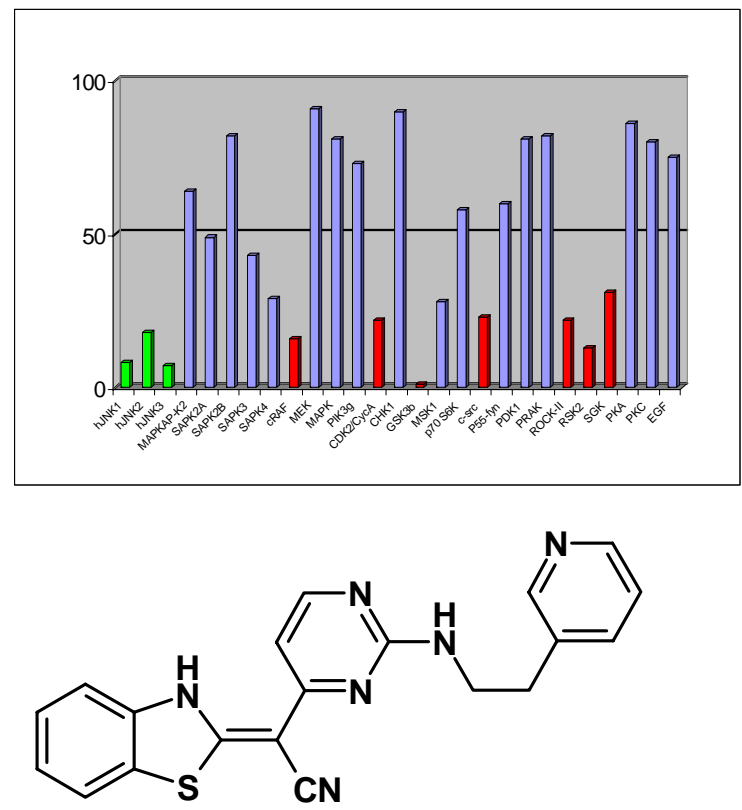

AS601245
Chart B

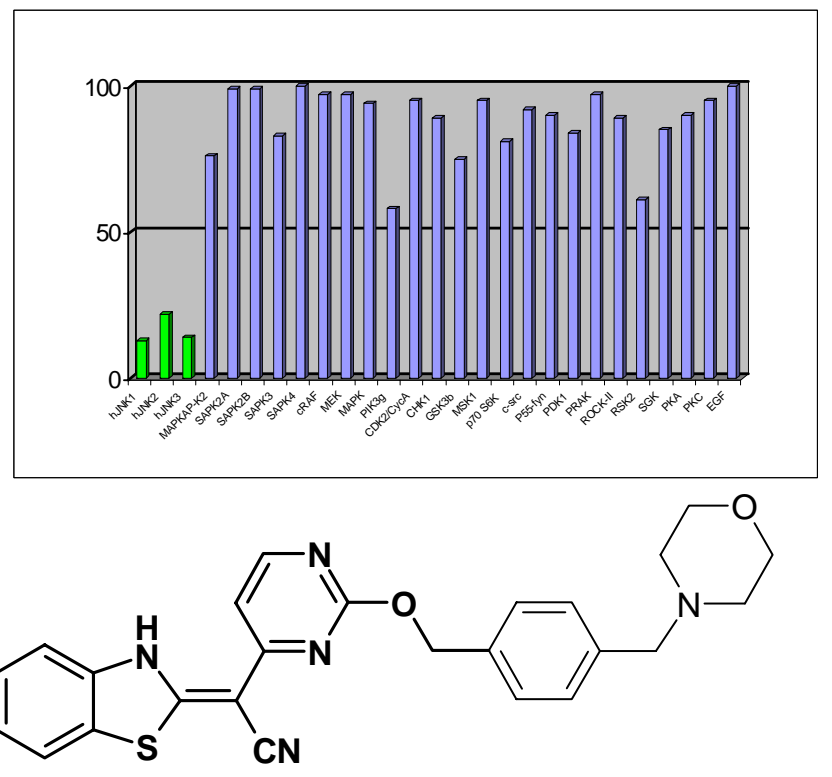

AS602801

Figure 3

Kinase Selective Profile

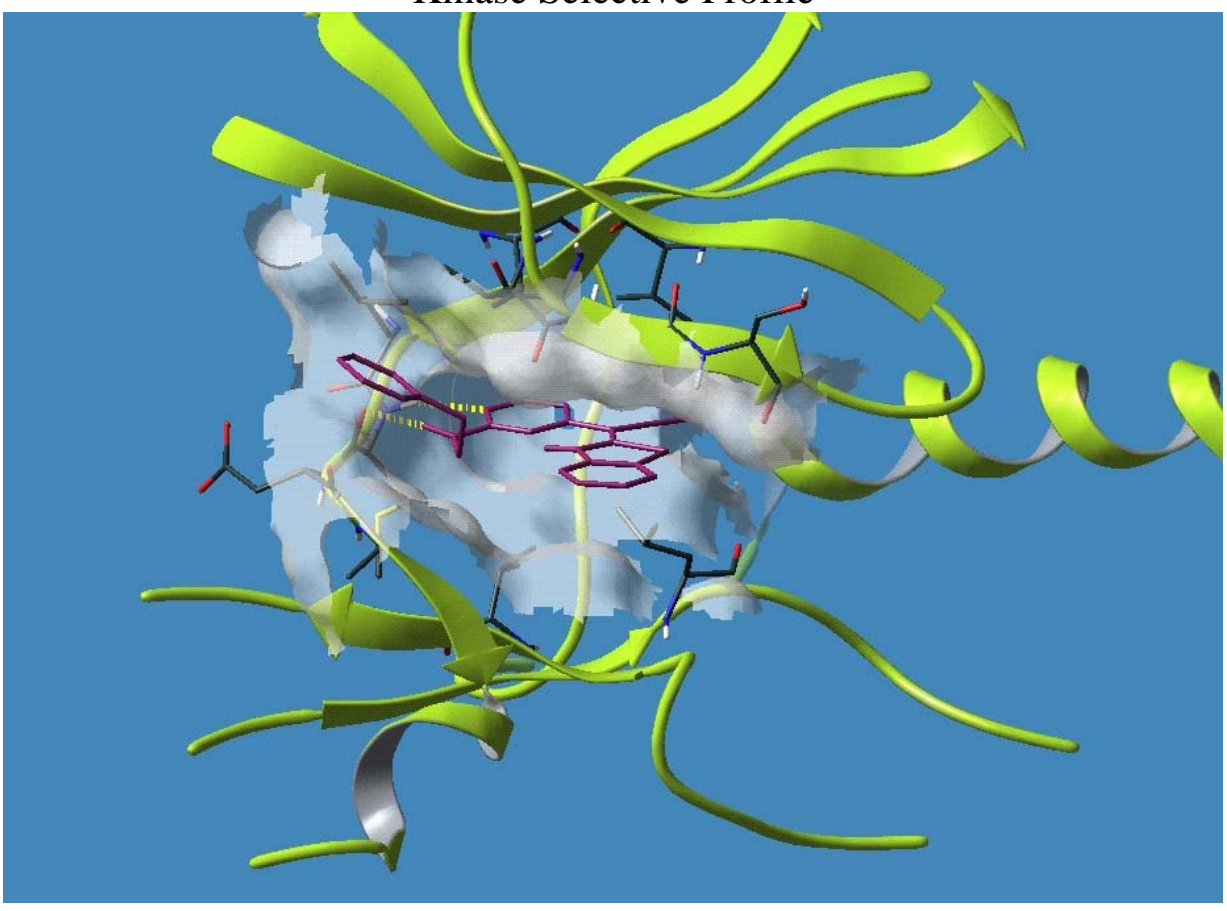

Figure 4 
Kinetic experiments at various ATP and inhibitor concentrations demonstrate that this compound is purely ATP competitive as shown by Lineweaver-Burk representation of Fig. 5.

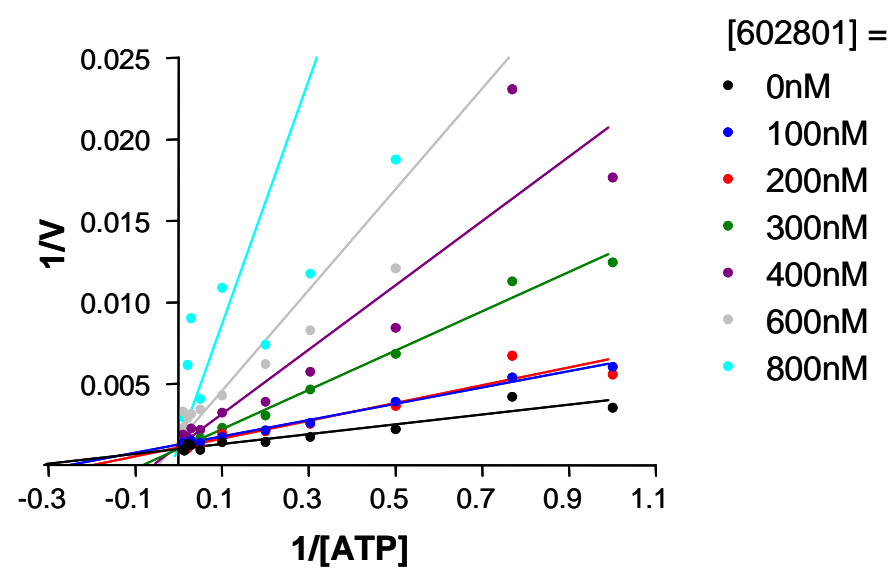

Figure 5

The best JNK inhibitors identified so far from this project are currently evaluated in preclinical studies, based on preliminary promising results in animal model of auto-immune diseases and neuronal apoptosis.

This example shows that it is possible to design new potent selective inhibitors of kinases, which are ATP competitive. The starting point is a generic kinase scaffold (hopefully proprietary) from which selectivity can be achieved through proper substitution driven by rationale design based on the kinase specific sequence and its 3D structure. This is a powerful tool, because it can be applied to different kinases, from the same central scaffold, providing proper information is available to direct chemists efforts in the right direction. Forthcoming kinases of therapeutic interest (among the 500 ones encoded by the human genome) will benefit from recent progresses in bioinformatics (in particular prediction of 3D structure based or primary sequence) and high-throughput production of protein constructs associated with new crystallisation technologies, which will provide new kinase 3D models or real structures for in silico design of selective inhibitors.

\section{Taking advantage of lack of selectivity}

The example described above shows how to transform a non-selective kinase inhibitor into a selective inhibitor with the help of computer-assisted drug design technologies based on the 3D structure of the chosen target kinase. Indeed, such starting chemical structures, build-up from new, proprietary scaffolds, have a much broader therapeutic potential since, they also inhibit other kinases; and therefore offer the potential to be transformed into selective inhibitors for these other kinases, by proper decoration and modification of the initial scaffold. Applying 
rationale design to explore these opportunities would represent a huge amount of work, which, in addition is not always feasible since 3D-structures don't exist for all kinases of interest. The solution we have chosen to explore this potential relies on combichem and consisted in the preparation of a library of 2,200 analogues of the initial non-selective inhibitor. This focused library was then tested against a panel of kinases selected from the ones for which activity was detected with the initial inhibitor as well as kinases of major interest in our therapeutic programs. By doing that we found plenty of non-selective inhibitors, but interestingly enough, we have also been able to identify small clusters of structurally-related compounds showing selective inhibition against each kinase selected, when tested at $10 \mu \mathrm{M}$ (Fig. 6).

\begin{tabular}{|l|c|c|c|c|c|c|c|c|}
\hline Compound & $\begin{array}{c}\text { CDK2cyclin } \\
\text { E(h) }\end{array}$ & cSRC(h) & GSK3(h) & Lck(h) & MSK1(h) & IKKb(h) & SGK(h) & AKT(h) \\
\hline AS249520 & 97 & 32 & 33 & 0 & 17 & 14 & 22 & 0 \\
\hline AS249234 & 0 & 94 & 3 & 81 & 12 & 1 & 29 & 12 \\
\hline AS247875 & 26 & 6 & 104 & 16 & 8 & 0 & 13 & 0 \\
\hline AS250669 & 0 & 25 & 2 & 100 & 16 & 11 & 4 & 8 \\
\hline AS250679 & 5 & 23 & 11 & 0 & 93 & 10 & 2 & 3 \\
\hline AS247859 & 19 & 32 & 29 & 27 & 12 & 98 & 14 & 43 \\
\hline AS249147 & 57 & 17 & 7 & 0 & 88 & 32 & 95 & 0 \\
\hline AS249215 & 9 & 5 & 41 & 8 & 0 & 4 & 9 & 92 \\
\hline
\end{tabular}

Figure 6

\section{Non-ATP competitive inhibitors}

An alternative way to avoid promiscuity in the design of kinase inhibitors is to look for noncompetitive inhibitors with respect to ATP, with the aim to hit interactions at kinase sites which are not commonly found within the enzyme class. Very little is known to really define a strategy based on rationale design for such type of inhibitors and therefore high-throughput screening of highly diverse collection of compounds remains as one of the most promising approach. This is the one we have followed as part of our efforts to find new MEK inhibitors as potential anticancer agents. Screening of 50K compounds led to the identification of a few positives among which AS701173 was characterised as a potent $\left(\mathrm{IC}_{50}=30 \mathrm{nM}\right)$ non ATP-competitive inhibitor of MEK-1. 
Lead optimisation based on this new selective MEK inhibitor and studies to understand their mechanism of action are in progress. Promising preliminary results have recently been obtained with newly found analogues demonstrating potent activity in tumour cell proliferation assays and in vivo models of cancer. Other non-competitive inhibitors of MEK have been reported as for example PD184352, which has also demonstrated anti-cancer potential. ${ }^{14}$

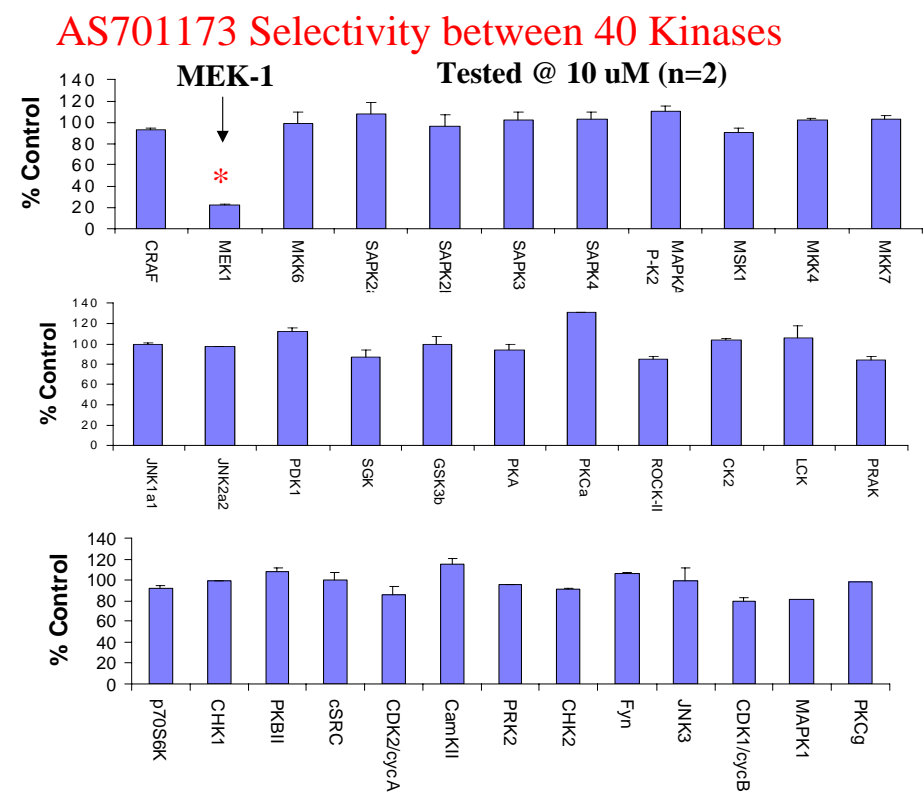

\section{Figure 7}

Evaluation of AS701113 against a panel of 40 available kinases show that this compound is highly selective for MEK since none of the other kinase was inhibited by more than $20 \%$ at $10 \mu$ M. (Fig. 7).

The identification of ATP non-competitive kinase inhibitors by screening is rather rare but offers the advantage to provide directly selective inhibitors ( since they interact with target kinase residues that are not shared with other kinases); the probability to find ATP competitive inhibitors by screening is much higher, but the strategy will very often require to follow-up with structure-based design work to transform initial Hits into selective compounds suitable to be developed as therapeutics.

\section{Kinases at the crossroad of complex signalling pathways}

Drug discovery is about matching chemical and biological spaces, by identifying validated and tractable targets (for a given disease) and drug-like molecules which are able to modulate them (Fig. 8). 


\section{Drug Discovery is about matching chemical and biological spaces}

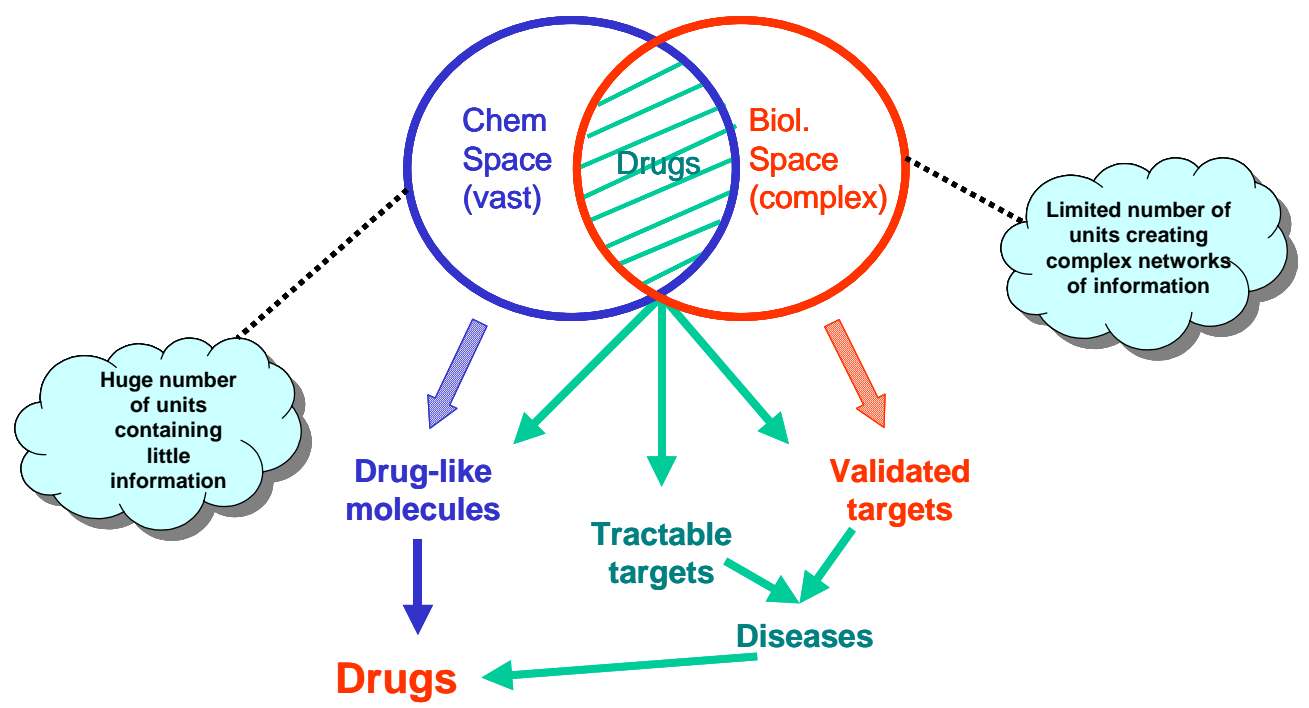

Figure 8

Chemistry space is characterized by diversity, [a very huge number of simple units (molecules)] while biology space is better characterized by complexity [limited number of units (proteins) creating extremely complex functional networks].

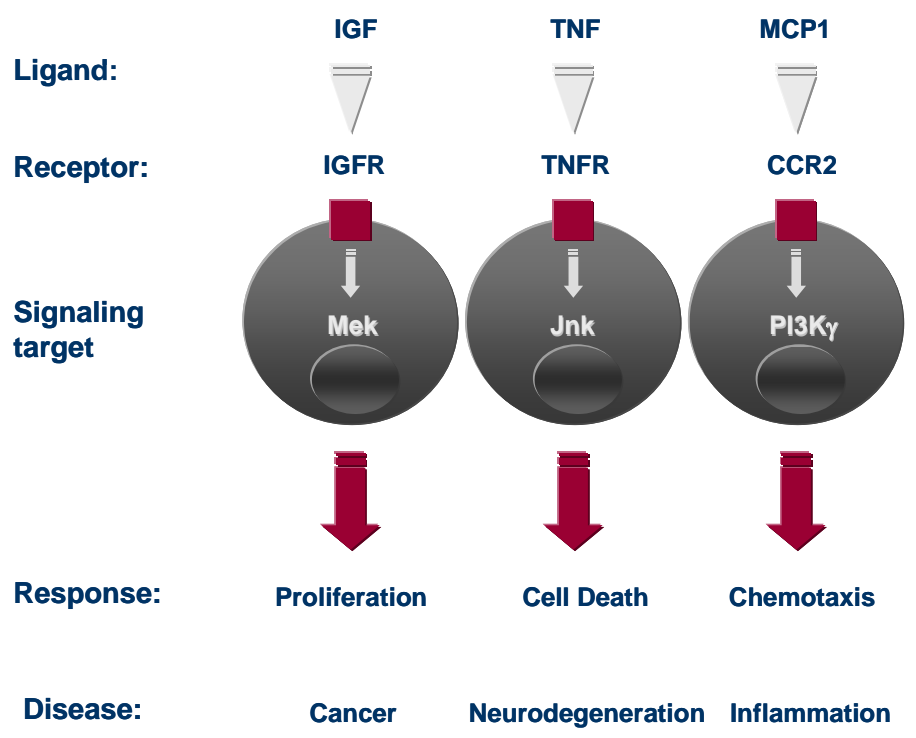

Figure 9 
Signal transduction is at the intersection of these 2 worlds, offering promising avenues to new drugs, in particular through kinases inhibition. In this area like in most drug discovery programs, chemists like to play with structural diversity and to apply SAR while focusing on a single identified target, and for intellectual comfort will reduce the biological complexity to its simplest expression as illustrated in Fig 9.

In this context, drug discovery efforts are concentrated on finding selective inhibitors of the selected kinase target, on the basis that such inhibitors will trigger clear functional responses, which will translate in a clear expected therapeutic effect.

Cell biologists and signalling experts will add pathways cross-talk complexity into the picture (as for example signalling events downstream of GPCR's as depicted in Fig.10) which has revealed the pleiotropic effects of many protein kinases involved in multiple signal transduction pathways. This is an invaluable contribution to kinases-based drug discovery which helps understand the mode of action of kinase inhibitors, their therapeutic potential, and possible side-effects, but will also contribute in a major way in the design of better compounds or identification of better targets (down stream kinases for example) for drug development.

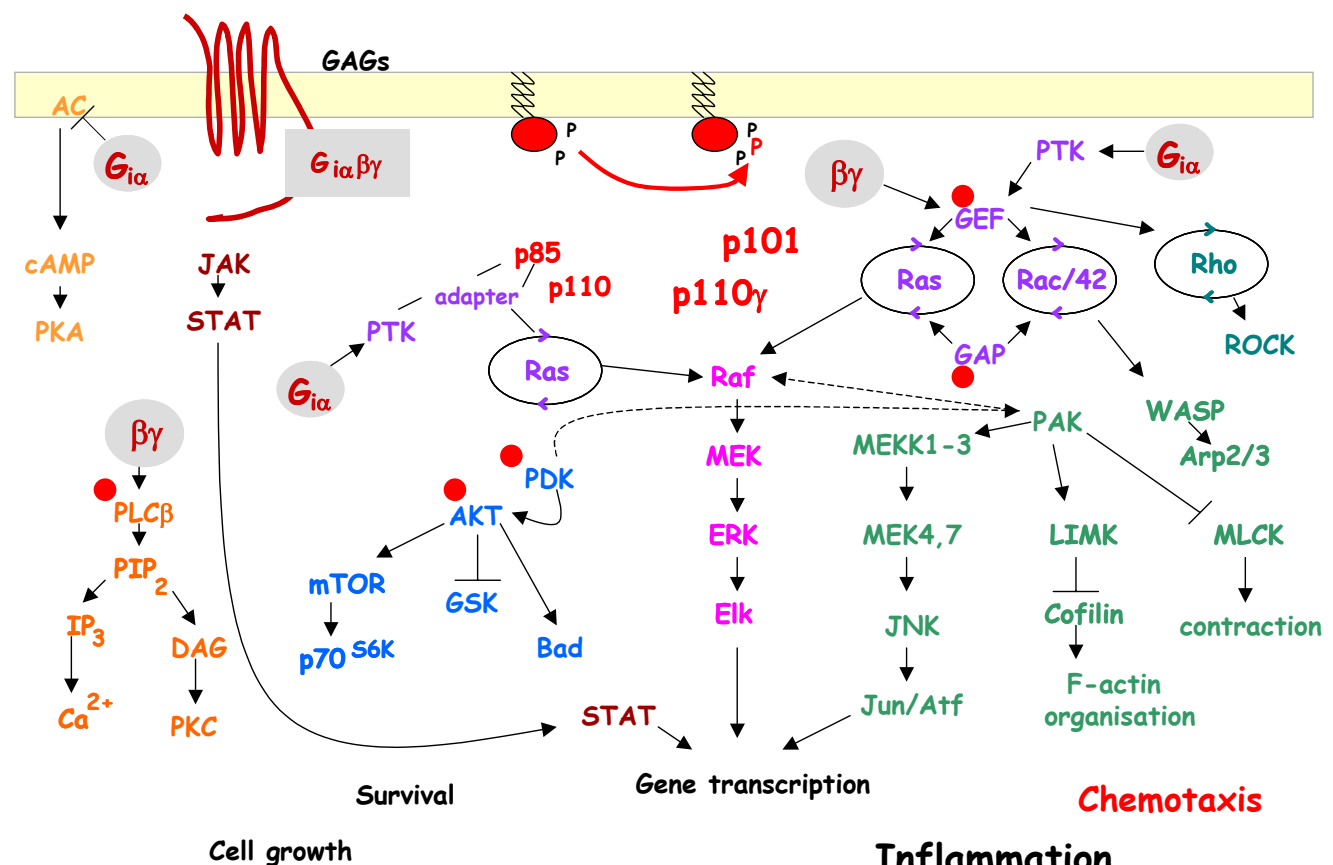

\section{Figure 10}

Recent progresses in proteomics and RNAi technologies give us now access to another level of complexity which is to understand the functional organisation of the full proteome (as for example in yeast ${ }^{15}$ ) or a selected part of the proteome (for example the human TNF- $\alpha / \mathrm{NF \kappa B}$ signal transduction pathway ${ }^{16}$ ) by mapping protein interaction network around known components of the investigated field and combining it with loss-of-function analysis. This 
approach is applicable to the study of any therapeutically relevant signal transduction process and will offer plenty of new relevant, validated targets in the future, together with deep knowledge of their functional role.

Other new emerging technologies ${ }^{17}$ integrating biochemistry, genetics and proteomics will help mining for protein kinase substrates (as for example the P-target ASKA technology described above) which is today a poorly understood and weakly documented area. Identification of new protein kinase substrates will pave the ways to discover safer and more efficient drugs by focusing on the best possible target for a given disease.

Having all these tools in hand, and all these experimental data available, the next step will be to understand and predict how the whole cell machinery will be affected when perturbating selectively one of the player of the complex intracellular network (with a kinase inhibitor for example) and to be able to modelise such a perturbation in a dynamic way, under different conditions (cell type, pathological versus non pathological, stress or not stressed, etc.). This is

the purpose of systems biology, ${ }^{18,19}$ which will provide in the future systems-level insight into mechanism-based drug discovery.

At the level of drug design, it means that we will move from rationale "structure-based" design of target-based selective inhibitors to the design of compounds that will induce a selected perturbation of cell regulation in a pathological state predicted to be beneficial for the patient. There is still a long way to go, but there is no doubt that kinase inhibitors will be at the heart of this promising and exciting new avenue in drug discovery.

\section{References}

1. Cohen, Ph. Nature Rev, Drug Discovery, 2002, 1, 309.

2. Graves, J. D.; Krebs, E. G. Pharmacology and Therapeutics, 1999, 82, 111.

3. Hunter, T. Signaling, 2000 and Beyond, Cell, 2000, 1000, 113.

4. Blume-Jensen, P.; Hunter, T. Nature, 2001, 411, 355.

5. Bridges, J. Chem Rev, 2001, 101, 2541.

6. Capdeville, R.; Buchdunger, E., Zimmermann, J.; Matter, A. Nature Rev Drug Discovery, 2002, 1, 493.

7. Manning, G.; Whyte, D. B.; Martinez, R.; Hunter, T. Science, 2002, 298, 1912.

8. Bishop, A. C.; Buzko, O.; Shokat, K. M. Trends in Cell Biology, 2001, 11, 167.

9. Bishop, A. C.; Kung, C.; Shah, K.; Witucki, L.; Shokat, K. M.; Liu, Y. J. Am. Chem. Soc., 1999, 121, 627.

10. Shah, K.; Shokat; K. M. Chemistry \& Biology, 2002, 9(1), 35.

11. Shokat, K.; Velleca, M. Drug Discovery Today, 2002, 7(16), 872.

12. Gotteland, J. P.; Rückle, T.; Biamonte, M.; Grippi-Vallotton, T.; Arkinstall, S.; Cambet, Y.; Camps, M.; Chabert, C.; Church, D.; Halazy, S.; Jiang, X.; Martinou, I.; Nichols, A.; Sauer, W. Journal of Medicinal Chemistry, 2005, 48, 4596. 
13. Carboni, S.; Hiver, A.; Szyndralewiez, C.; Gaillard, P.; Gotteland, J. P.; Vitte, P. A. Journal of Pharmacology and Experimental Therapeutics, 2004, 310, 25.

14. Sebolt-Leopold, J. S.; Dubley, D. T.; Herrera, R.; Becelaere, K. V.; Wiland, A.; Gowan, R. C.; Tecle, H.; Barrett, S. D.; Bridges, A.; Przybranowski, S.; Leopold, W. R.; Saltiel, A. R. Nature Medicine, 1999, 5(7), 810.

15. Gavin, A. C.; Bösche, M.; Krause, R.; Grandi, P.; Marzioch, M.; Bauer, A.; Schultz, J.; Rick, J. M.; Michon, A. M.; Cruciat, C. M.; Remor, M.; Höfert, C.; Schelder, M.; Brajenovic, M.; Ruffner, H.; Merino, A.; Klein, K.; Hudak, M.; Dickson, D.; Rudi, T.; Gnau, V.; Bauch, A.; Bastuck, S.; Huhse, B.; Leutwein, C.; Heurtier, M. A.; Copley, R. R.; Edelmann; A.; Querfurth, E.; Rybin, V.; Drewes, G.; Raida, M.; Bouwmeester, T.; Bork, P.; Seraphin, B.; Kuster, B.; Neubauer, G.; Superti-Furga, G. Nature, 2002, 415, 141.

16. Bouwmeester, T.; Bauch, A.; Ruffner, H.; Angrand, P-O.; Bergamini, G.; Croughton, K.; Cruciat, C.; Eberhard, D.; Gagneur, J.; Ghidelli, S.; Hopf, C.; Huhse, B.; Mangano, R.; Michon, A-M.; Schirle, M.; Schlegl, J.; Schwab, M.; Stein, M. A.; Bauer, A.; Casari, G.; Drewes, G.; Gavin, A-C.; Jackson, D. B.; Joberty, G.; Neubauer, G.; Rick, J.; Kuster, B.; Superti-Furga, G. Nature Cell Biology, 2004, 6(2), 97.

17. Bentos, B. K.; Rimmel, C.; Vellica, M.; Pasquali, C. Current Proteomics, 2004, 1, 83.

18. Kitano, H. Science, 2002, 295, 1662.

19. Davidov, E. J.; Holland, J. M.; Marple, E. W.; Naylor, S. Drug Discovery Today, 2003, 8(4), 175. 